\title{
Phase transition of potassium superoxide $\mathrm{KO}_{2}$
}

Sanghyun Lee $^{1}$, Shuki Torii ${ }^{1}$, Yoshihisa Ishikawa ${ }^{1}$, Masao Yonemura ${ }^{1}$, Takashi Kamiyama ${ }^{1}$

${ }^{1}$ Institute Of Materials Structure Science, KEK, 319-1106, Tokai, Japan

E-mail: lee@post.j-parc.jp

Magnetism is one of fundamental phenomena in condensed matter physics and material science. Generally, this magnetic property is found where unpaired electron occupies in $d$ - and f-orbital system of inorganic materials. For instance, magnetism of transition metal oxide with unpaired electron in d-orbital that gives fruitful physical phenomena through interplay between magnetism and other physical properties in superconductivity, colossal magnetoresistance(CMR), multiferroic, etc.

Alkali superoxide $\mathrm{AO2}(\mathrm{A}=\mathrm{K}, \mathrm{Rb}, \mathrm{Cs})$ is, so far, rare example of $\mathrm{p}$-orbital quantum molecular magnet that attract researchers whether well-known d-orbital physics can be applicable or not $[1,2]$. Two oxygen forms pure 02 molecular dumbbell through covalent bonding between $2 \mathrm{p}$-orbitals. Next, ionic bonding between $\mathrm{O} 2-$ and $\mathrm{A}+$ induces crystallization of $\mathrm{AO} 2$. This crystallization gives one more electron into $\Pi^{*}$ anti-bonding states of $\mathrm{O} 2$ molecule that induces quantum magnetism where one unpaired electron occupied in p-orbital inorganic system.

Due to relatively weak ionic bonding compared with covalent bonding of $\mathrm{O} 2$ molecular dumbbell, this $\mathrm{O} 2$ dumbbell rotation is main origin of structural phase transition, instead of $\mathrm{O} 2$ molecule deformation. It was reported that $\mathrm{O} 2$ dumbbell rotation induces six structural phases in $\mathrm{KO} 2[2,3]$. $\mathrm{KO} 2$ can be suitable materials for studying dumbbell rotation and $\mathrm{p}$-orbital magnetism.

Nevertheless, room temperature structure is unclear yet [3]. We measured temperature evolution from $4.6 \mathrm{~K}$ to room temperature using super-high-resolution neutron powder diffractometer(SuperHRPD) which beamline is installed in MLF, JPARC. We will discuss detail crystal structure.

[1] O. Volnianska and P. Boguslawski, J. Phys.: Condens. Matter 22, 073202 (2010)

[2] W. Känzig and M. Labhart, Journal de Physique Colloques 37, C7 (1976)

[3] M. Labhart et al., Phys. Rev. B 20, 53 (1978)

Keywords: Phase transition, $\mathrm{p}$-orbital magnetism, KO2 